\title{
The production of myco-diesel hydrocarbons and their derivatives by the endophytic fungus Gliocladium roseum (NRRL 50072)
}

Correspondence
Gary Strobel
uplgs@montana.edu

Received 10 July 2008

Revised 27 August 2008

Accepted 2 September 2008

\author{
Gary A. Strobel, ${ }^{1}$ Berk Knighton, ${ }^{2}$ Katreena Kluck, ${ }^{1}$ Yuhao Ren, ${ }^{1}$ \\ Tom Livinghouse, ${ }^{2}$ Meghan Griffin, ${ }^{3}$ Daniel Spakowicz ${ }^{3}$ and Joe Sears ${ }^{4}$ \\ ${ }^{1}$ Department of Plant Sciences, Montana State University, Bozeman, MT 59717, USA \\ ${ }^{2}$ Department of Chemistry, Montana State University, Bozeman, MT 59717, USA \\ ${ }^{3}$ Department of Molecular Biophysics and Biochemistry, Yale University, New Haven, CT 06520, \\ USA \\ ${ }^{4}$ Center for Lab Services/RJ Lee Group, 2710 North 20th Ave, Pasco, WA 99301, USA
}

\section{INTRODUCTION}

Endophytes are relatively unstudied microbes that exist in plants and cause no overt symptoms or signs of their presence. Their biological diversity, especially in temperate and tropical rainforests, is enormous. Many are known to produce biologically active substances and have found application in pharmacology (e.g. the anticancer drug taxol) and agriculture (Strobel \& Daisy, 2003). For instance, the recovery of a volatile antibiotic producing fungus, Muscodor albus, from Cinnamomum zeylanicum demonstrated that novel micro-organisms in the world's rainforests can be put to useful purposes (Worapong et al., 2001; Strobel et al., 2001; Strobel, 2006; Mercier \& Jimenez,

Abbreviations: PTR-MS, proton transfer mass spectrometry; SPME, solid-phase micro-extraction.
2007). This same fungus has been subsequently used as a selection tool to find other strains of M. albus (Ezra et al., 2004a). Surprisingly, a totally unrelated fungus, Gliocladium roseum (NRRL 50072), that grew from Eucryphia cordifolia (ulmo) stem samples resisted the volatile antibiotics of $M$. albus; this isolate of G. roseum itself produced volatiles with antibiotic properties (Stinson et al., 2003). Many volatile alkanes/alkenes were also produced by this fungus, including 4-decene, 9-methyl, 1octene and 1,3-octadiene along with a plethora of lowmolecular-mass esters, alcohols, ethers and fatty acids. Thus, it appeared that this organism possesses the metabolic machinery to produce high-energy-related hydrocarbons even under Petri plate conditions. Microbes producing such volatile high-energy substances are of extreme general interest given the world's general need for alternative fuel sources. 
Many microbes are known to produce various alkanes of chain length ranging from $\mathrm{C}_{16}$ to $\mathrm{C}_{35}$. Some microbes, such as photosynthetic bacteria, make hydrocarbons such as pristane and phytane, while cyanobacteria are well known as producers of 7- and 8-methylheptadecanes (Ladygina et al., 2006). Fungi, on the other hand, commonly make longchain hydrocarbons along with a series of low-molecularmass alcohols, ethers, esters, ketones and terpenoids (Sunesson et al., 1995). Wood-inhabiting fungi seem to make a wide range of volatile products (McAfee \&Taylor, 1999). The production of these volatile products by microbes is dependent upon the environmental conditions, the support medium and the microbial species being studied (Sunesson et al., 1995).

Many fungi are known to produce octane, 1-octene and lower-molecular-mass hydrocarbons (McAfee \& Taylor, 1999; Sunesson et al., 1995). Since G. roseum (NRRL 50072) produces a number of volatile hydrocarbons along with some corresponding oxygenated products (acids, esters, ethers and alcohols), it seemed important to determine if a modification of the environmental and cultural conditions of this organism would result in the production of more reduced compounds that are even more closely related to diesel fuel (Stinson et al., 2003). We now report that a genetically atypical G. roseum produces a wide range of hydrocarbons under conditions of limited oxygen on both oatmeal agar and a pure cellulose-based agar. Many of these hydrocarbons are some of the major substances in diesel fuel, including octane; 1-octene; heptane, 2-methyl; hexadecane; undecane, 4-methyl; nonane, 3-methyl; and benzene, 1,3-dimethyl. We term these volatiles of $G$. roseum 'myco-diesel'. This organism also makes other lipophilic compounds that are extractable from the aqueous phase. Collectively, these observations have important implications for fuel production with the help of a mycological resource. However, this report presents no information on the cost-effectiveness or other details to make $G$. roseum an alternative fuel source. Its ultimate value may reside in the genes/enzymes that control hydrocarbon production, and our paper is a necessary first step that may lead to development programmes to make this a commercial venture.

\section{METHODS}

Media selection and bioassay test. Pythium ultimum, mycelium only, was used to assay which medium supported the production of the highest concentrations of volatile bioactive substances using a previously described method (Strobel et al., 2001). Briefly, a $2.5 \mathrm{~cm}$ wide strip of agar was removed from the mid-portion of a standard Petri plate, creating two isolated halves of agar. Then, G. roseum was inoculated and grown on one side of the plate for 1,2 and 3 weeks to allow for production of volatile compounds. Finally, P. ultimum was inoculated onto the other half-moon agar piece on the opposite side of the plate, followed by sealing of the plate with a strip of Parafilm. The linear growth of $P$. ultimum was measured from the edge of the agar inoculum plug. The test on each medium was repeated at least three times and the standard deviations of the mean fungal growth were calculated.
The following media were assayed: (A) oatmeal agar (Difco); (B) corn starch $25 \mathrm{~g} \mathrm{l}^{-1}$; (C) glycerol $20 \mathrm{~g} \mathrm{l}^{-1}$ plus salts; (D) wheat stems $25 \mathrm{~g}$ $\mathrm{l}^{-1}$; (E) hay barley stems $25 \mathrm{~g} \mathrm{l}^{-1}$; (F) cellulose $25 \mathrm{~g} \mathrm{l}^{-1}$ plus salts; (G) cellulose $25 \mathrm{~g} \mathrm{l}^{-1}$ plus salts and yeast extract at $0.1 \mathrm{~g} \mathrm{l}^{-1}$; (H) cellulose $25 \mathrm{~g} \mathrm{l}^{-1}$ plus salts and $0.1 \mathrm{~g}$ soytone $\mathrm{l}^{-1}$; (I) cellulose $25 \mathrm{~g} \mathrm{l}^{-1}$ plus salts plus $0.1 \mathrm{mg} \mathrm{l}^{-1}$ of vitamins B1, B3 and B6; (J) potato starch $25 \mathrm{~g}$ $1^{-1}$; (K) potato dextrose (Difco) (PDA); and (L) Eucryphia cordifolia (ulmo) stem medium produced by boiling $30 \mathrm{~g}$ of newly developing stem tissues in water for $30 \mathrm{~min}$, filtering and then adding water to 1 litre. The salts and agar concentration used in each medium followed the recipe of the M1-D medium previously outlined by Pinkerton \& Strobel (1976). Each inhibition test was performed in triplicate and the data were analysed to obtain mean inhibition values and standard deviations.

Qualitative analysis of G. roseum gases. Gases were analysed in the air space above cultures of G. roseum grown for 18 days at $23{ }^{\circ} \mathrm{C}$ on agar slants under microaerophilic conditions in a $250 \mathrm{ml}$ brown glass bottle containing $100 \mathrm{ml}$ of the test agar and sealed with a top having a Teflon-based septum. These conditions were selected in order to provide a more reduced atmosphere to the culture, which could result in a greater number of hydrocarbons than observed in a Petri plate culture (Stinson et al., 2003). A prebaked solid-phase micro-extraction (SPME) syringe (Supelco) consisting of 50/30 divinylbenzene/carboxen on polydimethylsiloxane on a Stable Flex fibre was placed through a small hole drilled in the septum and exposed to the vapour phase for $45 \mathrm{~min}$. The syringe was then inserted into the splitless injection port of a Hewlett Packard 6890 gas chromatograph containing a $30 \mathrm{~m} \times 0.25 \mathrm{~mm}$ i.d. ZB Wax capillary column with a film thickness of $0.50 \mathrm{~mm}$. The column was temperature programmed as follows: $30{ }^{\circ} \mathrm{C}$ for 2 min increased to $220{ }^{\circ} \mathrm{C}$ at $5{ }^{\circ} \mathrm{C} \mathrm{min}^{-1}$. Details of all other aspects of running the gas analysis have been previously published (Strobel et al., 2007). Data acquisition and data processing were performed on the Hewlett Packard ChemStation software system. Initial identification of the unknowns produced by G. roseum was made through library comparison using the NIST database. All chemical names in this report follow the nomenclature of this database. In all cases, uninoculated control flasks were also analysed and the compounds found therein were subtracted from those appearing in the flask supporting fungal growth. Tentative identification of the fungal products was based on observed mass spectral data as compared to those in the NIST database. Final confirmatory identification was made for many of the compounds by comparing GC/MS data of authentic standards with the GC/MS data of the fungal products. The retention time of an authentic compound versus the fungal volatile was also measured and the relationship between the two is noted in the tables. The reported peak areas are relative values and are not strictly interchangeable, for comparison purposes, between tables. The experiments were repeated at least twice with comparable results.

Sourcing of organic compounds. Many of the hydrocarbons, organic alcohols, esters and acids were purchased from Aldrich/ Sigma. Some of the hydrocarbons were standard known compounds associated with diesel fuel obtained in Patagonia. This diesel source was also subjected to GC/MS and yielded such compounds as a complete series of alkanes starting with pentane through nonadecane. Numerous branched alkanes were also present, including heptane, 2methyl; nonane, 3-methyl; decane, 4-methyl; and many cyclohexane, naphthalene and benzene derivatives. The GC/MS data of some of these compounds were used for standard comparison purposes. Acetic acid, sec-octyl ester and 2-propanone, methylhydrazone were synthesized by standard organic methods (Hatzakis \& Smonou, 2005; Cavill et al., 2006).

Quantification of fungal volatiles. Proton transfer reaction-mass spectrometry (PTR-MS) was used to determine the concentration of 
volatiles produced by G. roseum (Ezra et al., 2004b; Bunge et al., 2008). Basically, the PTR-MS instrument ionizes organic molecules in the gas phase through their reaction with $\mathrm{H}_{3} \mathrm{O}^{+}$, forming mostly protonated molecules $\left(\mathrm{MH}^{+}\right.$, where $\mathrm{M}$ is the neutral organic molecule) which can then be detected by a standard quadrupole mass spectrometer. This process can be run on real air samples with or without dilution, since the primary constituents of air (nitrogen, oxygen, argon and carbon dioxide) have a proton affinity less than water and thus are not ionized. Most organic molecules (excepting alkanes) have a proton affinity greater than water and are therefore ionized and detected. A further advantage of PTR-MS is that from the known or calculated quantities, the reaction time, the amount of $\mathrm{H}_{3} \mathrm{O}^{+}$present, and the theoretical reaction rate constant for the proton transfer reaction, the absolute concentration of constituents in a sample can be quantified (Lindinger et al., 1998). Finally, an enormous advantage of PTR-MS is that it can be run in real time and continuously produce data on the concentrations of specific ions of interest.

Air-space analysis of the cultured and uninoculated samples was done by passing a small flow of air (medical-grade compressed air) through the culture bottles and then diluting this gas with catalytically scrubbed (to remove hydrocarbons) room air.

Measurements were made on an 18 day old culture of G. roseum grown at $23{ }^{\circ} \mathrm{C}$ on a $300 \mathrm{ml}$ oatmeal agar slant in a $950 \mathrm{ml}$ brown glass bottle with a septum that had been modified to possess both inlet and outlet tubes (Ezra et al., 2004b). The tests were run with a drift tube temperature of $30{ }^{\circ} \mathrm{C}$ with $10 \mathrm{ml} \mathrm{min}{ }^{-1}$ of air-space air being diluted into $90 \mathrm{ml} \mathrm{min}{ }^{-1}$ of hydrocarbon-free room air. The $1 / 10$ dilution kept the measurements within the linear dynamic range of the instrument and prevented water from condensing in the sample lines. The sample lines were constructed entirely from PFA Teflon tubing and fittings. Mass spectral scans were acquired from 20 to $250 \mathrm{Da}$. A minimum of 10 mass spectral scans was obtained for each sample.

Concentrations derived from the PTR-MS measurements were calculated using equations derived from reaction kinetics and assume that a reaction rate coefficient of $2 \times 10^{-9} \mathrm{ml} \mathrm{s}^{-1}$ is appropriate for all compounds (Lindinger et al., 1998; Ezra et al., 2004b). This method provides a simple means by which the measured ion intensity at any mass can be expressed as an equivalent concentration. In the event that a particular ion can be ascribed to a singular compound, then the concentration of that specific compound can be determined using the same procedure as above followed by correction for any product ion fragmentation. The product ion distribution is determined from mixtures prepared from pure standards.

Quantification of the amount of volatile hydrocarbons produced by G. roseum was performed by two different methods. The first is based on the total ion response as measured by PTR-MS. This total reflects only the contribution from those species having proton affinities greater than that of water and was determined from the total product ion intensity sum. It is important to note that impurity and reagent ions, which include $\mathrm{m} / z$ 21, 30, 32, 37-39, 46 and 55, were excluded from this sum.

The second method involves quantifying a single component and then scaling the concentrations of the other components that were detected by GC/MS by simply associating peak intensity with concentration as a first approximation of total gas concentration. In the present study, the information from the GC/MS experiments was used to identify the ion at $\mathrm{m} / z 159$ as acetic acid heptyl ester (Table 1). Although another compound, namely octanoic acid, methyl ester, also contributes to the intensity of this ion peak, its contribution is negligible since so little of it was present in the total gas mixture (Table 1). The product ion distribution was confirmed by separately running a standard sample and yielded the following ion fragments and relative abundances for the acetic acid heptyl ester: 43 (11\%), 57 (44\%), 61 (31\%), 99 (3\%) and 159 (11\%). The PTR-MS measurements were made twice with comparable results. Limitations of this indirect method result from the differential adsorption of volatiles to the SPME fibre and the differential ion efficiencies of the volatiles. Nevertheless, an estimate of the quantities of the various volatile components could be obtained.

Other fungal lipids. 'Bio-diesel' is a term that suggests a biological source of compounds that can effectively be used to run a diesel engine. Commonly, these compounds are primarily long-chained fatty acids derived from the hydrolysis of di- and triglycerides produced by higher plants and algae. Thus, in order to determine if any of these non-volatile lipids are produced by G. roseum in culture, the organism was grown for 30 days, with shaking, at $23{ }^{\circ} \mathrm{C}$ in 1 litre of medium $\mathrm{G}$ (in a 2 litre flask). The yeast extract used in the medium had been thoroughly extracted at least twice with methylene chloride in order to remove lipids. After incubation, the fungus was removed by filtration and the liquid extracted with two equal volumes of methylene choride and taken to dryness by flash evaporation at $40{ }^{\circ} \mathrm{C}$. TLC was performed, employing standard compounds as references in the following solvent systems: A, chloroform/methanol $(9: 1, \mathrm{v} / \mathrm{v}) ; \mathrm{B}$, toluene/ethyl acetate $(8: 2, \mathrm{v} / \mathrm{v})$; and $\mathrm{C}$, methylene chloride/methanol $(6: 4, v / v)$. Compounds were detected after spraying with the anisaldehyde/vanillin and sulfuric acid reagent (Stahl, 1969; Cardellina, 1991). Similar methods were applied to an identical medium that had not been inoculated with the fungus, as a control.

\section{RESULTS AND DISCUSSION}

\section{G. roseum NRRL 50072, the subject of this study}

This isolate of Gliocladium was obtained from Northern Patagonia as an endophyte of Eucryphia cordifolia (ulmo) (Stinson et al., 2003). Its species identity was initially determined based on its physical characteristics. On PDA, the fungal colony turned reddish after 10 days. The mycelium produced verticillate conidiophores possessing whorls of phialides $12-15 \mu \mathrm{m}$ in length. The conidia were hyaline, one-celled and ovoid, and borne in slimy protruding masses at the termini of the phalides. The conidia were smooth-walled and 3.0-3.5 $\times 4.5-6.0 \mu \mathrm{m}$. The collective morphological characteristics of this organism and the reddish pigment associated it most closely to Gliocladium roseum (Chen \& Huang, 2004). The organism was further characterized via molecular techniques on two separate occasions with identical results. Its 5.8S, ITS1 and ITS2 regions were isolated, cloned, partially sequenced and deposited in GenBank as AY219041. In addition, the partial $18 \mathrm{~S}$ rDNA sequence was entered as AY219040 (Stinson et al., 2003). In a BLAST search, as a close relative, Ascocoryne cylichnium appears with a coverage of $90 \%$ and an identity of $98 \%$. On the other hand, Ascocoryne sarcoides had a coverage of $89 \%$ and an identity of $99 \%$. Comparative molecular genetics, via a phylogenetic tree, indicates that $A$. sarcoides seems to be more closely related to this fungus. $A$. sarcoides is an ascomycete whose anamorph does not have a listing of Gliocladium sp., but as Coryne trichophora (Seifert, 1989). The telomorphs of Gliocladium spp. are generally considered as Nectria spp. and Hypocrea spp. (Hanlin, 1989). Nevertheless, it is distinctly possible that 
Table 1. GC/MS air-space analysis of the volatile compounds produced by $G$. roseum after 18 days incubation under microaerophilic conditions at $23^{\circ} \mathrm{C}$ on medium A (oatmeal agar)

Compounds found in the control oatmeal agar bottle are not included. Comparative GC/MS data/notes with standard compounds are indicated in the footnotes under 'Stds'. The total dry weight of the mycelial mat under these conditions was $38.9 \mathrm{mg}$.

\begin{tabular}{|c|c|c|c|c|}
\hline Time (min) & Relative area & Stds $s^{*} \ddagger$ & Possible compound $\S$ & Mol. mass (Da) \\
\hline 1.603 & 5.4 & $\ddagger$ & Oxirane, ethyl- & 72.06 \\
\hline 2.081 & 3.4 & ${ }^{*} \dagger$ & Heptane, 2-methyl-\$ & 114.14 \\
\hline 2.666 & 14.1 & $* \dagger$ & Octane $\$$ & 114.14 \\
\hline 3.138 & 15.5 & ${ }^{*} \dagger$ & 1-Octene\$ & 112.13 \\
\hline 4.598 & 24.5 & * & Ethanol & 46.04 \\
\hline 4.872 & 1.2 & * & Cyclohexene, 4-methyl- & 96.09 \\
\hline 5.204 & 13.5 & $\ddagger$ & Hexane, 2,4-dimethyl- (possible isomer) & 114.14 \\
\hline 5.378 & 10.8 & & Undecane, 2,6-dimethyl- & 184.22 \\
\hline 5.533 & 13.5 & $\ddagger$ & Hexadecane $\$$ (possible isomer) & 226.27 \\
\hline 5.941 & 10.5 & & Heptane, 5-ethyl-2,2,3-trimethyl- (or isomer) & 170.20 \\
\hline 6.365 & 8.7 & $\dagger$ & Undecane, 4-methyl-\$ & 170.20 \\
\hline 6.418 & 5.4 & & Heptane, 5-ethyl-2,2,3-trimethyl- (or isomer) & 170.20 \\
\hline 6.668 & 6.1 & & Octane, 3-ethyl-2,7-dimethyl- & 170.20 \\
\hline 6.768 & 12.1 & & Decane, 2,2,6-trimethyl- & 184.22 \\
\hline 6.931 & 6.8 & $* \dagger$ & Undecane\$ & 156.19 \\
\hline 7.112 & 5.4 & & Decane, 3,3,5-trimethyl- & 184.22 \\
\hline 7.173 & 6.4 & * & Nonane, 3-methyl-\$ & 142.17 \\
\hline 7.232 & 7.3 & * & 1-Propanol, 2-methyl- & 74.07 \\
\hline 7.325 & 10.0 & & Furan, 4-methyl-2-propyl- & 124.09 \\
\hline 7.481 & 6.7 & & Undecane, 4,4,-dimethyl- & 184.22 \\
\hline 7.648 & 13.3 & * & 1-Butanol, 3-methyl-, acetate & 130.10 \\
\hline 7.836 & 18.3 & * & 2-n-Butyl furan & 124.09 \\
\hline 8.026 & 10.8 & $\dagger$ & Benzene, 1,3-dimethyl- $\$$ & 106.08 \\
\hline 8.114 & 12.2 & & Decane, 3,3,5-trimethyl- & 184.22 \\
\hline 8.303 & 11.4 & * & Pentane, 1-iodo- & 197.99 \\
\hline 8.364 & 8.7 & * & 2-Hexanol & 102.10 \\
\hline 8.498 & 7.4 & * & Acetic acid, pentyl ester & 130.10 \\
\hline 8.735 & 14.9 & * & Hexanoic acid, methyl ester & 130.10 \\
\hline 9.066 & 11.5 & * & 1-Butanol, 3-methyl- & 88.09 \\
\hline 9.302 & 8.1 & & Phenol, 4-ethyl- & 122.07 \\
\hline 9.817 & 12.2 & * & 3-Octanone & 128.12 \\
\hline 10.054 & 11.4 & * & Acetic acid, hexyl ester & 144.12 \\
\hline 10.708 & 5.4 & * & 2-Heptanol & 116.12 \\
\hline 10.985 & 14.9 & & 7-Octen-2-one & 126.10 \\
\hline 11.242 & 12.1 & & Cyclopropane, propyl- & 84.09 \\
\hline 11.329 & 9.5 & * & Acetic acid, sec-octyl ester & 172.15 \\
\hline 11.545 & 17.9 & * & Acetic acid, heptyl ester & 158.13 \\
\hline 11.775 & 7.4 & * & Octanoic acid, methyl ester & 158.13 \\
\hline 11.938 & 9.5 & & 3,5-Octadiene $(Z, Z)$ & 110.11 \\
\hline 12.127 & 9.4 & * & 2-Octanol & 130.14 \\
\hline 12.878 & 9.4 & * & Acetic acid & 60.02 \\
\hline 12.931 & 22.7 & * & Acetic acid, octyl ester & 172.15 \\
\hline 13.381 & 4.1 & & 3-Octen-2-ol ( $Z)$ & 128.12 \\
\hline 13.584 & 7.3 & & Neoisolongifolene & 204.19 \\
\hline 14.455 & 8.8 & * & Propanoic acid, 2-methyl- & 88.05 \\
\hline 14.543 & 8.8 & & Cycloheptanone, 2-methylene- & 124.09 \\
\hline 14.651 & 12.9 & & 1H-Indene, octahydro-, cis- & 124.13 \\
\hline 14.926 & 8.1 & & Cyclopentadiene, 5,5-dimethyl-1-ethyl- & 122.11 \\
\hline 15.335 & 10.1 & * & Butyrolactone & 86.04 \\
\hline 15.477 & 8.1 & * & Acetic acid, decyl ester & 200.18 \\
\hline 15.673 & 13.5 & & Pentanoic acid, 3-methyl- & 116.08 \\
\hline 15.835 & 6.8 & & Cyclodecene & 138.14 \\
\hline 16.472 & 12.7 & & Pentanoic acid & 102.07 \\
\hline
\end{tabular}


Table 1. cont.

\begin{tabular}{|lccll|}
\hline Time (min) & Relative area & Stds ${ }^{\star} \dagger \neq$ & \multicolumn{1}{c|}{ Possible compound\$ } & Mol. mass $($ Da) \\
\hline 17.653 & 16.9 & $\star$ & Hexanoic acid & 116.08 \\
18.360 & 13.6 & $\star$ & Phenylethyl alcohol \\
19.588 & 8.0 & $*$ & Phenol, 4-ethyl-2-methoxy- & 122.07 \\
& & & & 152.08 \\
\hline
\end{tabular}

${ }^{\star}$ Denotes that the retention time and MS spectrum closely matched or were identical to an authentic standard compound. Those compounds without a designated footnote have a mass spectrum that most closely matched the appropriate compound in the NIST database.

$\dagger$ Denotes that the compound, as detected in diesel fuel, was used as a standard.

\#Indicates that the retention time of the standard compound does not match that of the fungal product. It is to be noted that sometimes retention times of a compound can be strongly influenced by other compounds in the injection mixture. Alternatively, the designated compound may represent an isomer of that compound.

\$Denotes that the compound was detected in Patagonian diesel.

this organism, as an atypical Gliocladium roseum, may be an anamorph of Ascocoryne spp. The organism is deposited at the NRRL as 50072 and in the living mycological collection at Montana State University as 2259.

\section{Fungal inhibitory properties and gas production of $\mathbf{G}$. roseum on different media}

Of the various media tested for inhibition of P. ultimum (A-L; see Methods), the two demonstrating the best activity were A (oatmeal agar) and $G$ (cellulose-based medium).

Preliminary GC/MS analysis of the volatiles produced in Petri plate culture, with media A, G, K and L, showed a wide mixture of various lipids, alcohols, esters, acids and ketones (Stinson et al., 2003 and data not shown). Based on these observations, these media were selected for growth and gas production by G. roseum, especially under restricted oxygen availability in order to increase the content of hydrocarbons and their derivatives in the air space above the cultures. In addition, medium $\mathrm{L}$ was selected for study since it is composed entirely of the host extract. As such, it may be possible to learn if the host can supply enough critical ingredients to the fungus to produce hydrocarbons.

\section{Production of volatile hydrocarbons and their derivatives by $\mathbf{G}$. roseum under microaerophilic conditions on the oatmeal-based medium $\mathbf{A}$}

Gas analysis of the fungus grown in microaerophilic conditions on medium A revealed more than twice the number of volatiles as in the air space over the fungus grown in Petri plates on medium A or medium $\mathrm{K}$ under normal conditions (Stinson et al., 2003). Of these volatiles, many are commonly found in diesel fuel. Some of the detected volatiles included the following: 1-octene; octane; hexadecane; heptane, 2-methyl; undecane, 4-methyl; nonane, 3-methyl; benzene, 1,3-dimethyl; and cyclohexene, 4methyl; these were confirmed with standards. Other alkanes were also detected in the air space of this organism, including undecane, 2,6-dimethyl; decane, 3,3,5-trimethyl; octane, 3-ethyl-2,7-dimethyl; decane, 3,3,6-trimethyl; undecane, 4,4-dimethyl; and others (Table 1). These more complex branched alkanes were identified based upon the mass spectral data reported in the NIST database. Most interesting, however, was the appearance of a series of straight-chained alkane acetyl esters including those of pentyl, hexyl, heptyl, octyl, sec-octyl and decyl alcohols (Table 1). Each derivative alkane of these alcohols is a major ingredient in diesel fuel (data not shown). One of the most abundant compounds in the volatile phase was the acetic acid heptyl ester and it was used in the quantification of the other components. Many free alcohols were also detected in the air space of G. roseum; these included 2-octanol; ethanol; 1-propanol, 2-methyl; 1butanol, 3-methyl; 2-heptanol; and phenylethyl alcohol (Table 1). Also present was a group of free fatty acids including acetic acid; propanoic acid, 2-methyl; pentanoic acid; pentanoic acid 3-methyl; and hexanoic acid. Other oxygenated compounds present in the air space of the culture included some furans, ketones and a lactone. One of the most unusual substances found in the fungal volatiles was pentane, 1-iodo. This compound appeared in the air spaces of both the bottles and the Petri plates of medium A (Table 1). Phenylethyl alcohol and sesquiterpenoids such as neoisolongifolene are commonly found as volatile products of the endophytic Muscodor spp. (Strobel, 2006).

\section{Production of hydrocarbons and their derivatives by $\mathbf{G}$. roseum under microaerophilic conditions on the cellulose-based medium $\mathbf{G}$}

The dramatic increase in global food prices spurs the need to sever the biofuel market from food production. An attractive carbon source is cellulose, the world's most abundant natural organic compound. Cellulose is digestible by many rainforest fungi, including those closely related to G. roseum, such as Trichoderma spp. G. roseum grew for several months on a plate of medium $G$ and it 
remained inhibitory to $P$. ultimum. Gas analysis after 18 days of incubation on medium $G$ showed the presence of a number of hydrocarbons commonly found in diesel fuel in the air space of this culture. These included heptane, octane and dodecane (Table 2). Other hydrocarbons were also present including cyclopentane, 1,1,3,3-tetramethyl; hexane, 3,3-dimethyl; and decane, 2,6,7-trimethyl. The alkane esters present in the gases above cultures on the oatmeal-based medium A were not found in the volatiles from medium G; however, there were a number of alkanebased free alcohols including 1-decanol, 1-butanol, 2hexanol and 1-heptanol. Furthermore, a number of other oxygenated hydrocarbons appeared as ketones such as 3hexanone; 3-hexanone, 4-methyl; and pentanone, 4methyl, which was the most abundant volatile product in this mixture (Table 2). Each of these volatile hydrocarbons is readily combustible. Both ethanol and acetic acid were present in medium $G$ as they were in medium $A$ along with some sesquiterpenoids including aciphyllene, eremophilene, and others (Tables 1 and 2).

This fungus does make other lipids in addition to the hydrocarbons appearing in the gas phase. For instance, the methylene chloride extract of medium G, after 30 days of incubation, yielded at least $130 \mathrm{mg}$ of lipoidal substances. TLC in solvents A, B and C, with cochromatography of appropriate standards, indicated the presence of linoleic acid, linolenic acid combined with a mixture of triglycerides, and one or more cholic acid derivatives. Only trace amounts of lipoidal compounds were detected in the control by comparable methods. This suggests that, in addition to the volatiles of this fungus being important and useful, so too are components in the aqueous phase.

\section{Production of hydrocarbons and their derivatives by $\mathbf{G}$. roseum under microaerophilic conditions on the ulmo-based medium $\mathbf{L}$}

Medium L, made from extract of the the host plant of $G$. roseum, was tested for the production of volatiles, to determine if the host plant from which the fungus was originally isolated could support both fungal growth and simultaneous production of a volatile mixture containing alkanes or other hydrocarbons. It was surmised that the microaerophilic conditions in the test conditions would mimic those that this endophytic fungus may experience in the host tissue in terms of nutrient and oxygen availability, since endophytes are located in the intercellular spaces of plant cells. Interestingly, several alkanes and an alkene (diesel hydrocarbons) were detected in the volatiles in the air space of the 18 day old culture, including octene; undecane, 2,7-dimethyl; decane, 4-methyl; and tridecane (Table 3). Numerous other alkanes were also produced, along with several sesquiterpenoids (Table 3 ). Since the volatiles of this fungus possess antibiotic properties, these compounds may play a role in protecting the plant from invading pathogens (Ezra et al., 2004b).

\section{Quantification of the volatile products of $\mathbf{G}$. roseum}

The total concentration of volatile organic compounds produced by the fungus in medium A and detectable by PTR-MS was estimated to be in the order of 80 p.p.m.v. with the contribution of the control agar gases being excluded (Fig. 1). This value was calculated by summing up the concentration from each ion in the mass spectrum (Fig. 2 ). Ions that were deemed as reagent ions, contaminant ions such as $\mathrm{O}_{2}^{+}$or $\mathrm{NO}^{+}$, or water-related ions, were not included in this calculation. Additional major contributors to the total volatiles were methanol, ethanol and acetaldehyde ions, making up about $30-40 \%$ of the total. Also, major contributors were ions at $\mathrm{m} / z 89$ and 101. The identity of these two ions could not be established on the basis of the compounds shown in Table 1, but the ion at $\mathrm{m} / \mathrm{z} 89$ is consistent with protonated butyric acid ion. Overall, it seems that compounds in addition to those shown in Table 1 are also produced by this fungus. This is not unexpected given the fact that the SPME fibre (Stable Flex) does not absorb all volatile compounds. Additional experiments are required to fully characterize the entire suite of volatiles produced by this organism.

Also, in order to quantify the concentration of the hydrocarbons, and their derivatives, present in the air space of medium A, an indirect method involving PTR-MS was used. One of the most abundant volatiles is acetic acid heptyl ester, with a retention time of $11.545 \mathrm{~min}$ (Table 1) and its protonated molecule is detected at $\mathrm{m} / z 159$ by the PTR-MS. The concentration of the acetic acid heptyl ester was quantified assuming that all of the peak at $\mathrm{m} / z 159$ originated from this compound. The peak concentration for this compound was in the order of 500 p.p.b.v. Assuming that the other volatile compounds are present in the air-space vapour in the same proportions as determined by the GC/MS, then the concentration of the acetic acid heptyl ester can be used to estimate the concentrations of all other ingredients in the volatile phase (Table 1). The total concentration of volatiles in the air space of medium A was estimated at 4 p.p.m.v. It is to be noted, however, that certain volatile compounds may not have adsorbed to the fibre and others may have adsorbed in a non-uniform manner, thus yielding a lower than expected total amount of material in the gas phase as calculated by this indirect method versus the concentration of volatile compounds based on total ion yield (above). In addition, the concentration of volatiles found in medium $G$ was calculated by this indirect method and shown to be in the range of $50 \%$ of that in medium A.

\section{Origins of fungal hydrocarbons and crude oil}

Two biochemical pathways in micro-organisms known for the production of straight-chained hydrocarbons are of chief interest in this study since these compounds are among the main ingredients of diesel fuel. These are the 'elongation-decarboxylation' and the 'head-to-head 
Table 2. GC/MS air-space analysis of the volatile compounds produced by $\mathrm{G}$. roseum after 18 days incubation under microaerophilic conditions at $23{ }^{\circ} \mathrm{C}$ on medium $\mathrm{G}$ (cellulose-based)

Compounds found in the control bottle are not included in this table. Comparative GC/MS data/notes with standard compounds are indicated in the footnotes under 'Stds'. The total dry weight of the mycelial mat under these conditions was $4.7 \mathrm{mg}$.

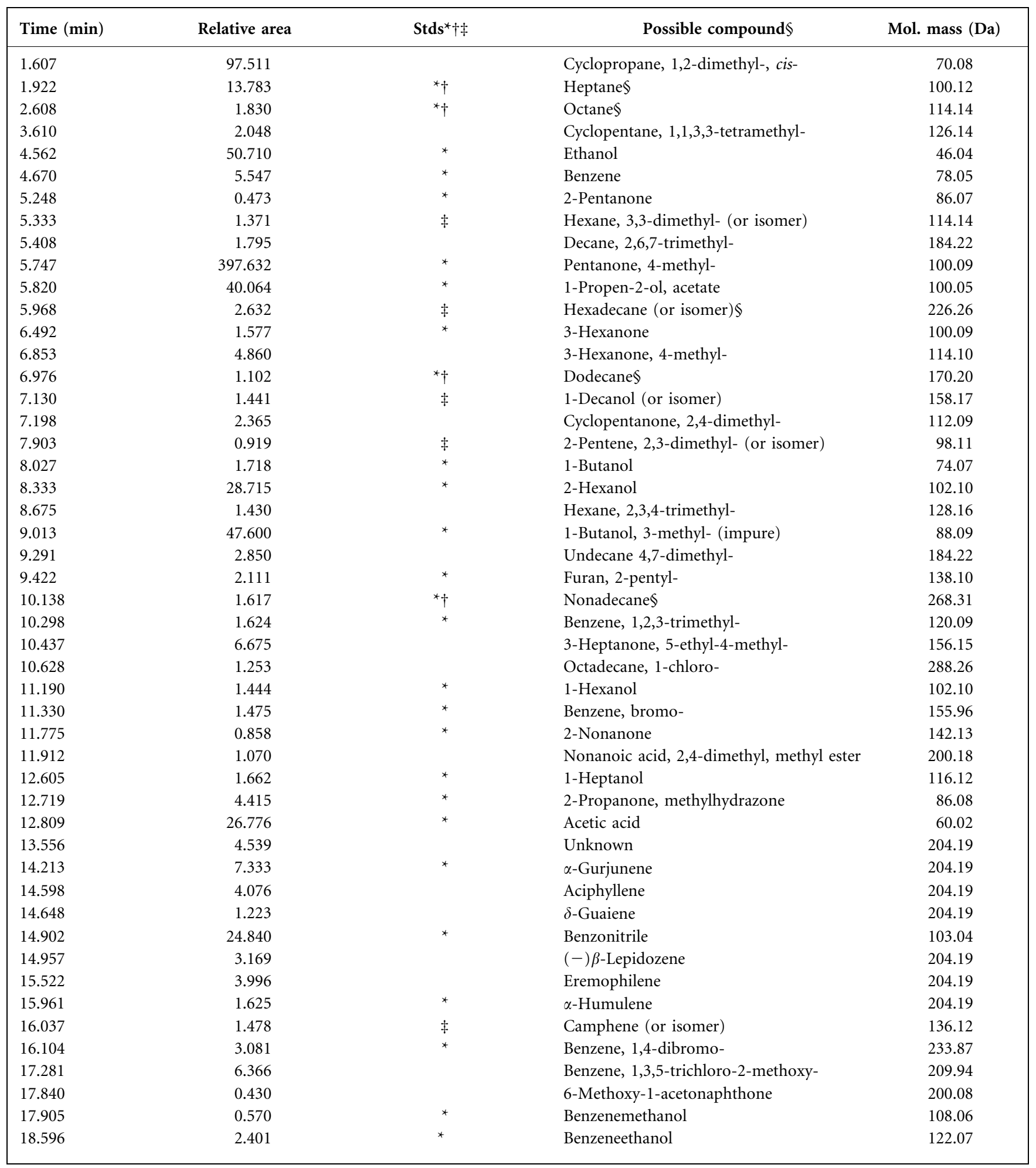

$*, \dagger, \ddagger$, See Table 1 for details. 
Table 3. GC/MS air-space analysis of the volatile compounds produced by $\mathrm{G}$. roseum after 18 days incubation under microaerophilic conditions at $23{ }^{\circ} \mathrm{C}$ on medium $\mathrm{L}$ (ulmo medium)

Compounds found in the control bottle are not included in this table. Comparative GC/MS data/notes with standard compounds are indicated in the footnotes under 'Stds'. The total dry weight of the mycelial mat under these conditions was $5.3 \mathrm{mg}$.

\begin{tabular}{|c|c|c|c|c|}
\hline Time $(\min )$ & Relative area & Stds ${ }^{\star} \dagger \neq$ & Possible compound $\S$ & Mol. mass (Da) \\
\hline 1.552 & 14.136 & * & 1-Butene, 2-methyl- & 70.08 \\
\hline 1.816 & 0.743 & * & Heptene & 100.12 \\
\hline 2.806 & 2.304 & $* \dagger$ & 1-Octene\$ & 112.12 \\
\hline 3.572 & 0.992 & & Furan, 2-methyl- & 82.04 \\
\hline 4.385 & 5.389 & & 1,3-Cyclopentadiene, 1,2-dimethyl- & 94.08 \\
\hline 4.789 & 1.071 & $\dagger$ & Dodecane, 4,6-dimethyl- $\$$ & 198.23 \\
\hline 4.850 & 1.933 & & Undecane, 4,6-dimethyl- & 184.22 \\
\hline 5.369 & 3.074 & & Undecane, 5-methyl- & 170.20 \\
\hline 6.110 & 0.845 & & Nonane, 5-propyl- & 170.20 \\
\hline 6.171 & 5.051 & $\dagger$ & Undecane, 4-methyl- $\$$ & 170.20 \\
\hline 6.246 & 2.394 & & Undecane, 2,7-dimethyl- & 184.22 \\
\hline 6.396 & 2.879 & + & Decane, 4-methyl- $\$$ & 156.19 \\
\hline 6.546 & 1.447 & & 1-Decane, 8-methyl- & 154.17 \\
\hline 8.407 & 2.114 & * & 1-Butanol, 2-methyl- & 88.09 \\
\hline 8.625 & 0.984 & ${ }^{*} \dagger$ & Tridecane\$ & 184.22 \\
\hline 9.484 & 1.314 & & Dodecane, 2,7,10-trimethyl- & 212.25 \\
\hline 11.591 & 11.384 & * & Benzene, 1-methoxy-2-methyl- & 122.07 \\
\hline 12.075 & 1.046 & * & Benzene, 1-methoxy-3-methyl- & 122.07 \\
\hline 12.847 & 2.816 & & 1,3-cyclopentadiene, 1,2,3,4,5-pentamethyl- & 136.12 \\
\hline 14.292 & 4.192 & & Phenol, 3-ethyl- & 122.07 \\
\hline 14.770 & 2.669 & & Pinocarveol & 152.12 \\
\hline 14.927 & 0.900 & * & $(+)$-Aromadendrene & 204.19 \\
\hline 15.367 & 0.871 & * & $\alpha$-Humulene & 204.19 \\
\hline 16.324 & 2.829 & * & Myrtenol & 152.12 \\
\hline 16.691 & 1.006 & & Benzene, 1,3,5-trichloro-2-methoxy- & 209.94 \\
\hline 20.292 & 0.817 & $\ddagger$ & Phenol, 4-ethyl- & 122.07 \\
\hline
\end{tabular}

*, †,

condensation' pathways discussed by Ladygina et al. (2006). The latter pathway seems most likely to be involved in the formation of hydrocarbons in G. roseum. This pathway would necessarily involve such precursors as acetyl-CoA and malonyl-CoA condensing to increase chain length, and undergoing other reactions such as decarboxylation, decarbonylation and hydration as well as oxidation-reduction, to yield both odd- and even-numbered hydrocarbons. Other precursors, such as L-methylmalonylCoA, may also be involved in the hydrocarbon synthetic pathways of G. roseum since branched hydrocarbons are produced. Still other pathways, yet to be elucidated, may account for the doubly substituted carbons in the branched alkanes that are produced by G. roseum (Tables 1 and 2). Finally, in the case of the alkane ester series, an esterification reaction would occur between acetic acid and the alkane alcohol, resulting in the alkane ester series being produced by this fungus (Table 1). Consistent with this is the presence of a relatively large amount of acetic acid and also free fatty alcohols. G. roseum must also possess other lipid synthase enzymes since numerous terpenoids are found in its gas phase (Tables 1,2 and 3).

Certainly, it is both timely and interesting that G. roseum can utilize cellulose for the production of hydrocarbons given the enormous volumes of foodstuff grains currently being utilized for alcohol (fuel) production. However, the yields of these compounds were lower than those found on the oatmeal-based medium, probably because the digestion of cellulose is rate limiting. Increases in the yields of these products may be enhanced by new developments in fermentation technology, membrane technologies and genetic manipulation (Danner \& Braun, 1999). Of some concern, however, is an anomaly in the production of hydrocarbons by G. roseum. For instance, when G. roseum was originally isolated and studied, [8]annulene was the most abundant compound in the gas phase (Stinson et al., 2003). After 7 years of storage at $-70{ }^{\circ} \mathrm{C},[8]$ annulene was not detected under any conditions in any GC/MS experiment. This phenomenon may be attributed to some 


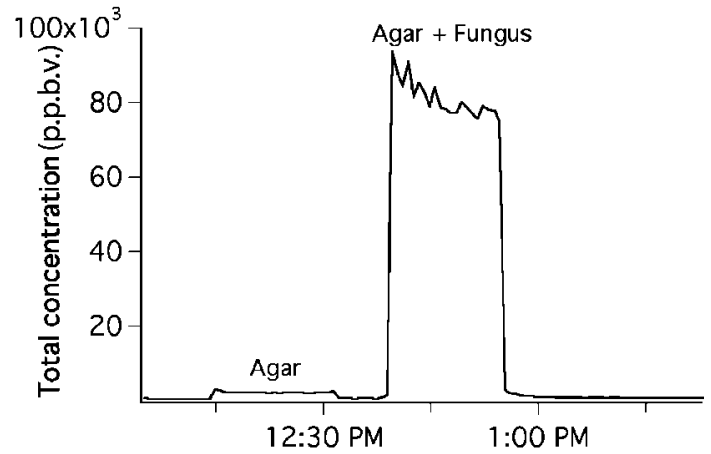

Fig. 1. Total ion production as measured by PTR-MS in the air space of an 18 day old culture of $G$. roseum on oatmeal-based medium (medium A). The ions produced from the agar alone are shown on the left while those of the fungus plus the agar are on the right. lons that were deemed reagent ions, contaminant ions such as $\mathrm{O}_{2}^{+}$or $\mathrm{NO}^{+}$, or water-related ions were not included in this calculation.

genetic instability of the organism under prolonged conditions of storage, or through attenuation after successive subculturing on nutrient-rich media.

This work has interesting implications in consideration of the processes that contribute to the formation of crude oil. Most geologists view crude oil and natural gas as products arising from the compression and heating of ancient organic substances over the course of geological time. In view of this work, perhaps it is not unreasonable to speculate that some hydrocarbons in the earth's upper mantle may have arisen via the fermentation of plant

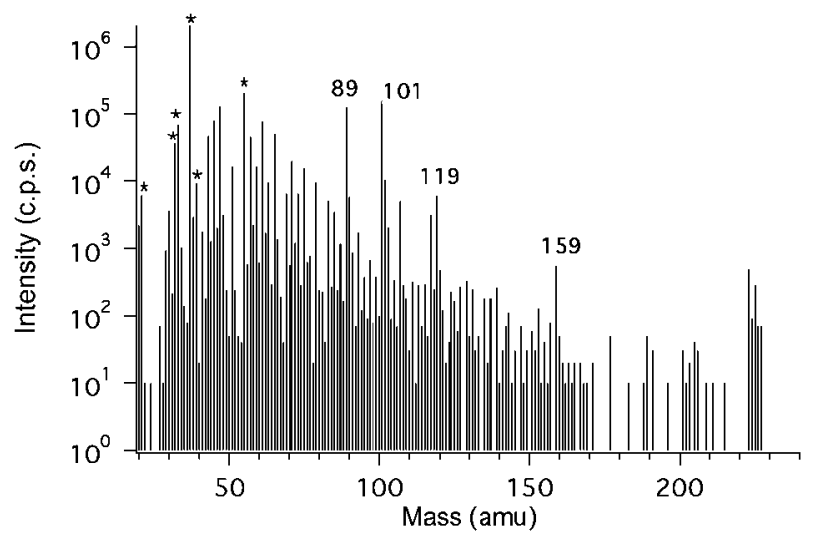

Fig. 2. PTR-MS mass spectrum generated from the air space over an 18 day old culture of $G$. roseum grown at $23 \mathrm{C}$ on medium $\mathrm{A}$. Prominent ions associated with the volatile components produced by $G$. roseum were identified. Peaks marked with an asterisk represent primary or impurity reagent ions, $\mathrm{H}_{3} \mathrm{O}^{+}\left(\mathrm{H}_{2} \mathrm{O}\right)_{n}, \mathrm{NO}^{+}$, $\mathrm{O}_{2}^{+}$, which were not included in the calculation of the total gas concentration. The ions observed at masses over $200 \mathrm{amu}$ (atomic mass units or daltons) are associated with the agar medium. materials by fungi under conditions of limited oxygen. The endophytic microbes may be the first ones involved in the disintegration and utilization of plant material since, by definition, they are located in the plant tissues at the time of the demise of the plant. Also, the crude soluble ingredients of the host plant may serve as precursor compounds for the production of hydrocarbons (Table 3). Finally, given the uniqueness of the observation that $G$. roseum can make such important fuel-based compounds, including fatty acids, from a cellulose-based medium, it would seem that a much larger search for such organisms in our natural environment needs to be launched.

\section{ACKNOWLEDGEMENTS}

The authors wish to acknowledge the financial assistance of NSF grant CBET-0802666, and the Montana Agricultural Experiment Station. Comments and suggestions on this report were kindly offered by Scott Strobel of the Department of Molecular Biophysics and Biochemistry at Yale University as well as Arny Demain of Drew University and David Light of the Connecticut C.I. Robison, Connecticut.

\section{REFERENCES}

Bunge, M., Araghipour, N., Mikoviny, T., Dunkl, J., Schnitzhofer, R., Hanzel, A., Schinner, F., Wisthaler, A., Margesin, R. \& Tilmann, M. D. (2008). On-line monitoring of microbial volatile metabolites by proton transfer reaction-mass spectroscopy. Appl Environ Microbiol 74, 2179-2186.

Cardellina, J. H. (1991). HPLC separation of taxol and cephalomannine. J Liq Chromatogr 14, 659-665.

Cavill, J., Elliott, R. L., Evans, G., Jones, I. L., Platts, J. A., Ruda, A. M. \& Tomkinson, N. O. (2006). The $\alpha$-effect in iminium ion catalysis. Tetrahedron 62, 410-421.

Chen, J. T. \& Huang, J. W. (2004). Identification of Gliocladium roseum, the causal agent of brown spot of kind oyster mushroom Pleurotus eryngii. Plant Pathol Bull 13, 17-26.

Danner, H. \& Braun, R. (1999). Biotechnology for the production of commodity chemicals from biomass. Chem Soc Rev 28, 395-405.

Ezra, D., Hess, W. M. \& Strobel, G. A. (2004a). New endophytic isolates of Muscodor albus, a volatile-antibiotic-producing fungus. Microbiology 150, 4023-4031.

Ezra, D., Jasper, J., Rogers, T., Knighton, B., Grimsrud, E. \& Strobel, G. A. (2004b). Proton-transfer reaction-mass spectroscopy as a technique to measure volatile emissions of Muscodor albus. Plant Sci 166, 1471-1477.

Hanlin, R. T. (1997). Illustrated Genera of Ascomycetes. St Paul, MS: American Phytopathological Society.

Hatzakis, N. S. \& Smonou, I. (2005). Asymmetric transesterification of secondary alcohols catalyzed by feruloyl esterase from Humicola insolens. Bioorg Chem 33, 325-337.

Ladygina, N., Dedyukhina, E. G. \& Vainshtein, M. B. (2006). A review on microbial synthesis of hydrocarbons. Process Biochem 41, 10011014.

Lindinger, W., Hansel, A. \& Jordan, A. (1998). On-line monitoring of volatile organic compounds at pptv levels by means of protontransfer-reaction mass spectrometry (PTR-MS): medical applications, food control and environmental research. Int J Mass Spectrom Ion Process 173, 191-241. 
McAfee, B. J. \& Taylor, A. (1999). A review of the volatile metabolites of fungi found on wood substrates. Nat Toxins 7, 283-303.

Mercier, J. \& Jimenez, J. (2007). Potential of the volatile-producing fungus Muscodor albus for the control of building molds. Can J Microbiol 53, 404-410.

Pinkerton, F. \& Strobel, G. A. (1976). Serinol as an activator of toxin production in attenuated cultures of Helminthosporium sacchari. Proc Natl Acad Sci U S A 73, 4007-4011 (USA).

Seifert, K. A. (1989). Coryne trichophora, comb. nov., and the implications of its conidiomatal anatomy. Stud Mycol 31, 157-164.

Stahl, E. (1969). Thin Layer Chromatography: a Laboratory Handbook. Springer.

Stinson, M., Ezra, D., Hess, W. M., Sears, J. \& Strobel, G. A. (2003). An endophytic Gliocladium sp. of Eucryphia cordifolia producing selective volatile antimicrobial compounds. Plant Sci 165, 913-922.

Strobel, G. A. (2006). Muscodor albus and its biological promise. J Ind Microbiol Biotechnol 33, 514-522.
Strobel, G. A. \& Daisy, B. (2003). Bioprospecting for microbial endophytes and their natural products. Microbiol Mol Biol Rev 67, 491-502.

Strobel, G. A., Dirksie, E., Sears, J. \& Markworth, C. (2001). Volatile antimicrobials from Muscodor albus, a novel endophytic fungus. Microbiology 147, 2943-2950.

Strobel, G. A., Kluck, K., Hess, W. M., Sears, J., Ezra, D. \& Vargas, P. N. (2007). Muscodor albus E-6, an endophyte of Guazuma ulmifolia, making volatile antibiotics: isolation, characterization and experimental establishment in the host plant. Microbiology 153, 2613-2620.

Sunesson, A.-L., Vaes, W. H. J., Nilsson, C. A., Blomquist, G., Andersson, B. \& Carlson, R. (1995). Identification of volatile metabolites from five fungal species cultivated on two media. Appl Environ Microbiol 61, 2911-2918.

Worapong, J., Strobel, G. A., Ford, E. J., Li, J. Y., Baird, G. \& Hess, W. M. (2001). Muscodor albus anam. nov., an endophyte from Cinnamomum zeylanicum. Mycotaxon 79, 67-79.

Edited by: M. Tien 